\title{
A New Dynamic Transmission-Mode Selection Scheme for AMC/HARQ-Based Wireless Networks
}

\author{
Xiaohui Ma ${ }^{1,2,3}$, Guobing $\mathrm{Li}^{1,4}$ and Guomei Zhang ${ }^{1,4}$ \\ ${ }^{1}$ State Key Laboratory of Geo-information Engineering \\ Xi'an, Shaanxi 710054, P. R. China \\ [e-mail: shellmei.zhang@stu.xjtu.edu.cn] \\ ${ }^{2}$ Institute of Geography Spatial Information, Information Engineering University \\ Zhengzhou, Henan 450052, P. R. China \\ ${ }^{3}$ Xi'an Research Institute of Surveying and Mapping \\ Xi'an, Shaanxi 710054, P. R. China \\ ${ }^{4}$ Department of Information and Communication Engineering, Xi' an Jiaotong University \\ Xi'an, Shaanxi 710049, P. R. China \\ [e-mail: \{gbli,zhanggm\}@mail.xjtu.edu.cn] \\ *Corresponding author: Guobing Li
}

Received February 4, 2017; accepted July 6, 2017; published November 30, 2017

\begin{abstract}
In this paper, we study the cross-layer design for the AMC/HARQ-based wireless networks, and propose a new dynamic transmission-mode selection scheme to improve system spectrum efficiency. In the proposed scheme, dynamic thresholds for transmission-mode selection in each packet transmission and retransmission are jointly designed under the constraint of the overall packet error rate. Comparing with the existing schemes, the proposed scheme is inclined to apply higher modulation order at the first several (re)transmissions, which corresponds to higher-rate transmission modes thus higher average system spectrum efficiency. We also extend the cross-layer design to MIMO (Multi-input Multi-output) communication scenarios. Numerical results show that the proposed new dynamic transmission-mode selection scheme generally achieves higher average spectrum efficiency than the conventional and existing cross-layer design.
\end{abstract}

Keywords: Wireless networks, AMC, HARQ, Cross-layer design, MIMO

\footnotetext{
This research was supported by the Natural Science Foundation of China (NSFC) under Grant No. 61431011, the Natural Science Foundation of Shaanxi Province under Grant No. 2016JQ6007, and the State Key Laboratory of Geo-information Engineering of China under Grant No. SKLGIE2016-Z-2-3 and SKLGIE2016-Z-2-5.
} 


\section{Introduction}

D ue to the impact of large and small-scale fading, the actual wireless channel inherently shows strong time-varying characteristic, making link adaption a necessary and fundamental technique for wireless communications. Adaptive feedback communications were addressed as early as in 1968 and shows remarkable effectiveness in combating fading ${ }^{[1]}$. In 1972, a variable-rate transmission scheme was developed by dynamically adjusting the pulse durations to improve the transmission reliability ${ }^{[2]}$. However, due to the limit of hardware and computation capability, adaptive transmissions had not received sufficient attention until 1990s, when the OFDM (Orthogonal Frequency Division Multiplexing) technique was rediscovered, and multi-channel modulation as well as new channel estimation techniques were significantly advanced. So far, adaptive communication schemes have been intensely studied in order to improve the throughput and spectrum efficiency, or reduce the system complexity ${ }^{[3-5]}$. Nowadays, adaptive modulation and coding (AMC) and hybrid automatic repeat request (HARQ) are commonly used for link adaption in the physical-layer (PHY) and medium access (MAC) layer in modern wireless communication systems such as LTE (Long-Time Evolution), LTE-A (LTE-Advanced) and 5G (The 5th generation mobile networks) ${ }^{[6-8]}$. As key technologies to guarantee the reliability and spectrum efficiency in wireless communication systems, AMC adaptively select the modulation and coding parameters to improve the reliability and transmission rate according to the feedback of channel-state information (CSI). Meanwhile, HARQ ensures reliable transmissions by means of forward error correction (FEC) and retransmission using $\mathrm{ARQ}^{[9]}$.

Although AMC and HARQ are generally separated function entities for the PHY and MAC layer ${ }^{[--7,9]}$, numerous studies have revealed significant performance gains brought by cross-layer optimization and design in spectrum efficiency and transmission reliability ${ }^{[10-11]}$. Cross-layer design jointly controls parameters in different protocol layers in order to improve the overall system performance. For the physical and multiple-access layer, the transmission mode controlled by AMC, which corresponds to modulation order and channel coding, are usually optimized or designed jointly with the retransmission schemes controlled by ARQ/HARQ ${ }^{[12-18]}$. For example, in [12] and [13], the cross-layer designs for AMC and ARQ were investigated with or without the consideration of packet delay. In [14], a cross-layer discrete-time Markov chain based queuing model is developed to jointly exploit the capabilities of HARQ and AMC, in which the thresholds for the selection of transmission mode is designed to ensure the packet-error-rate (PER) requirement of the last retransmission to be met. In [15], a dynamic threshold for transmission mode selection was developed for the cross-layer design with AMC and HARQ. In [16] and [17], the cross-layer design for practical broadband communication systems were studied. Although the work above successfully mapped the QoS requirements from upper layers into PER requirements for the PHY and MAC layer, the optimal design to find every specific transmission mode in each packet (re)transmission remains open. To address this problem, the work in [18] provided a framework to approximate the whole PER through modulation coding rate for AMC and the retransmission times for HARQ, and proposed a dynamic threshold switching scheme. However, in [18] the PER requirements for each retransmission of a given packet are constrained to be identical, which may cause a performance loss in spectrum efficiency. The work in [19] and [20] for MIMO (Multiple-input Multiple output) communication systems revealed the potential performance gain of cross-layer design in MIMO case. 
In this work, we developed a new transmission-mode selection scheme for the cross-layer design of AMC/HARQ-based wireless transmissions. Using the existing PER approximation provided in [14], [18] and references therein, in this paper dynamic thresholds for transmission mode selection in each packet transmission and retransmission are jointly derived under the constraint of the PER tolerance of each specific packet. Compared with existing schemes such as [18], the main difference or contribution of this paper lies that, by taking advantage of the mechanism of retransmissions in HARQ, the dynamic thresholds are designed to favorite higher modulation and coding rate, which corresponds to higher-rate transmission modes at the first several (re)transmissions, which results in higher average system spectrum efficiency. Furthermore, in order to make our scheme more applicable, we extend the cross-layer design to MIMO communication scenarios.

The remainder of the paper is organized as follows: in Section 2, the system model for cross-layer design in AMC/HARQ based wireless transmission is introduced. In Section 3, the conventional and existing dynamic-threshold scheme for AMC/HARQ cross-layer design are discussed, then the new dynamic transmission mode selection mode is proposed and analyzed. Numerical results are provided in Section 4 and Section 5 concludes the paper.

\section{System Model}

The system block diagram of the cross-layer design in an AMC-HARQ based wireless network is shown in Fig. 1. Triggered by the QoS (Quality of Service) requirements from upper layers, the cross-layer design jointly controls AMC and HARQ by dynamically adjusting the maximum retransmission time $N_{\mathrm{r}}$ in the MAC layer and transmission mode (TM mode, corresponds to the modulation coding rate) in the PHY layer.

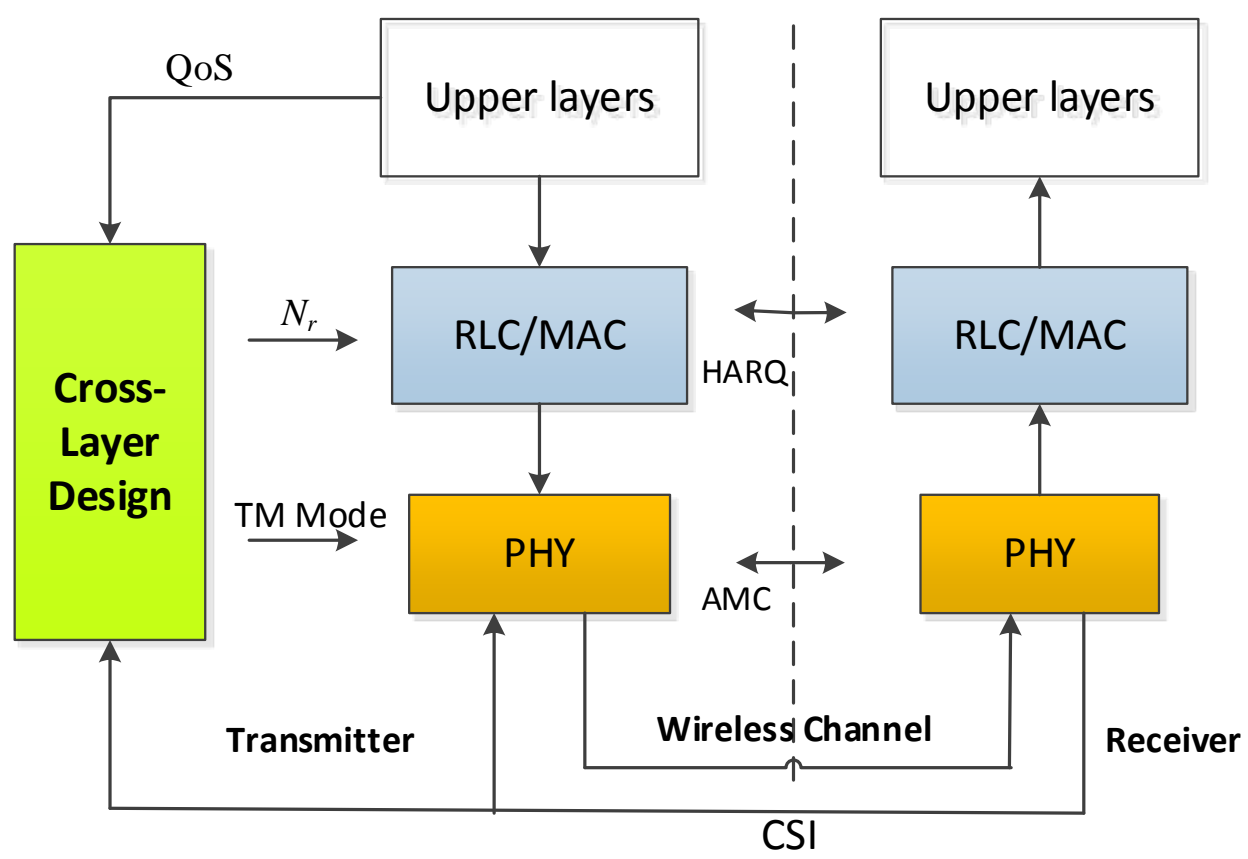

Fig. 1. System block diagram for corss-layer design with AMC and HARQ 
In the MAC/RLC layer, the transmitter determines whether it requires packet retransmission through the feedback information ACK (ACKnowledgment) or NAK (Negative AcKnowledgment). To ensure the transmission efficiency, the maximum retransmission times that HARQ allows is set to a constant number $N_{\mathrm{r}}$, which means that, the packet retransmission will be terminated anyway either when the packet is received successfully or failed to deliver after $N_{\mathrm{r}}+1$ times. In wireless systems such as LTE and LTE-A, the cross-layer design supports both chase-combing HARQ and incremental-redundancy HARQ $^{[7]}$.

In the physical layer, the transmitter selects appropriate transmission mode (TM), according to the channel condition. For example, in the LTE-A system, there are M+1 types of transmission mode. Besides the silence mode, the parameters of the rest of the transmission modes can be found in Table $\mathbf{1}^{[14][18]}$.

Table 1. Available transmission modes in the physical layer

\begin{tabular}{|c|c|c|c|c|c|}
\hline & TM 1 & TM 2 & TM 3 & TM 4 & TM 5 \\
\hline \hline $\begin{array}{c}\text { Modulation } \\
\text { coding rate }\end{array}$ & $\begin{array}{c}\text { BPSK } \\
1 / 2\end{array}$ & $\begin{array}{c}\text { QPSK } \\
2 / 3\end{array}$ & $\begin{array}{c}\text { QPSK } \\
5 / 6\end{array}$ & $\begin{array}{c}16 \text { QAM } \\
2 / 3\end{array}$ & $\begin{array}{c}16 \text { QAM } \\
5 / 6\end{array}$ \\
\hline$R_{n}($ bit/symbol) & $1 / 2$ & $4 / 3$ & $5 / 3$ & $8 / 3$ & $10 / 3$ \\
\hline$a_{n, 0}$ & 4447.4 & 2068.5 & 514.7 & 850.9 & 142.9 \\
\hline$a_{n, 1}$ & 2298.6 & 1344.3 & 3297.8 & 372.3 & 895.7 \\
\hline$a_{n, 2}$ & 5944.9 & 1428.4 & 7247.9 & 3567.3 & 1057.1 \\
\hline$g_{n, 0}$ & 11.104 & 3.315 & 1.759 & 0.816 & 0.339 \\
\hline$g_{n, 1}$ & 21.012 & 6.997 & 6.196 & 1.895 & 1.657 \\
\hline$g_{n, 2}$ & 34.203 & 10.569 & 10.224 & 3.378 & 2.706 \\
\hline$\gamma_{p_{n, 0}}(d B)$ & -1.212 & 3.623 & 5.502 & 9.172 & 11.660 \\
\hline$\gamma_{p_{n, 1}}(d B)$ & -4.337 & 0.127 & 1.164 & 4.946 & 6.131 \\
\hline
\end{tabular}

In this work, we consider a block-fading channel and assume that the wireless channel coefficients follow Nakagami- $m$ distribution. Hence, the PDF (Probability Dense Function) of the received SNR(Signal-to-Noise Ratio) can be expressed by:

$$
p_{\gamma}(\gamma)=\frac{m^{m} \gamma^{m-1}}{\bar{\gamma}^{m} \Gamma(m)} \exp \left(-\frac{m \gamma}{\bar{\gamma}}\right)
$$

where $\bar{\gamma}=E\{\gamma\}$ denotes the average received SNR, $\Gamma(m)$ is the gamma function and $m \geq 1 / 2$ represents the key characteristic of the Nakagami- $m$ channel. For $m=1$, the channel is degraded to Rayleigh distribution, which is widely used for the rich-scattering channel.

Thanks to the work in [14][18], the PER of all transmission modes when applying HARQ in the MAC layer can be approximated. For chase-combining HARQ (the Type-I HARQ), the packet error rate in the transmission mode $n$ at the $i$-th transmission time can be expressed by:

$$
\operatorname{PER}_{n, i}(\gamma) \approx \begin{cases}1, & 0 \leq \gamma<\frac{\gamma_{p_{n, 0}}}{i+1} \\ a_{n, 0} e^{-g_{n, 0}(i+1) \gamma}, & \gamma \geq \frac{\gamma_{p_{n, 0}}}{i+1}\end{cases}
$$


Similarly, when the PHY transmisson is combined with incremental-redundancy HARQ (Type-II HARQ), the packet error rate is:

$$
\operatorname{PER}_{n, i}(\gamma) \approx \begin{cases}1, & 0 \leq \gamma<\gamma_{p_{n, i}} \\ a_{n, i} e^{-g_{n, i} \gamma}, & \gamma \geq \gamma_{p_{n, i}}\end{cases}
$$

where $a_{n, i}, g_{n, i}$ and $\gamma_{p_{n, i}}$ are the variables to calculated PER in each transmission mode as listed in Table 1, where $n$ is index of the transmission mode, $i$ denotes the current transmission time and $i=0$ represents the first-time transmission.

It is noted that the PERs above are derived under the assumptions of infinite transmission buffer and error-free and delayless feedback channel for ACK/NAK, which are also made in this work.

\section{Cross-layer Design of AMC and HARQ}

\subsection{Transmission-mode Selection for Cross-layer Design with AMC and HARQ}

As depicted in Section 2, there are totally $M+1$ types of transmission mode corresponding to $M+1$ non-overlapped SNR region, which can readily be divided by increasing SNR thresholds $\left\{\gamma_{n}\right\}_{n=0}^{M+1}$ with $\gamma_{0}=0$ and $\gamma_{M+1}=+\infty$. For the instantaneous SNR $\gamma \in\left[\gamma_{n}, \gamma_{n+1}\right)$, the mode $n$ will be selected for packet transmission. That is, by adjusting the SNR thresholds $\left\{\gamma_{n}\right\}_{n=1}^{M}$, we can utilize different modulation type for AMC.

A conventional way to obtain $\left\{\gamma_{n}\right\}_{n=1}^{M}$ is to calculate $\gamma_{n}$ with the following inequality by treating each transmission the same as the first-time transmission, i.e.,

$$
\operatorname{PER}_{n, 0}(\gamma) \leq P_{\text {loss }}
$$

where $P_{\text {loss }}$ is the desired packet error rate which is determined the QoS requirement from upper layers. This is a straightforward and simple way to obtain (4) $\gamma_{n}$ since (4) only requires the PER in the first transmission to satisfies a certain threshold, while it ignores the difference in performance gain caused by the retransmission.

Substituting the expression of PER into (4), the SNR thresholds combined the Type-I and Type-II HARQ can be written as:

$$
\gamma_{n}=\frac{1}{g_{n, 0}} \ln \left(\frac{a_{n, 0}}{P_{\text {loss }}}\right), n=1,2, \ldots, M
$$

From (5), the transmission mode can be readily determined by the threshod $\gamma_{n}$. However, the conventional scheme above does not consider the retransmission gain brought to the system by HARQ. It can be noticed that when the time of (re)transmission increases, the PER curve of each model tends to be close to the low-SNR region, which means, for the same SNR, retransmission may take higher order mode of transmission.

Taking this into account, the basic idea of a dynamic threshold switching scheme was proposed in [18], which proposed that, all transmission should use different threshold, meaning that the PER of all transmission are required to meet the conditions below :

$$
\operatorname{PER}_{n, i}(\gamma) \leq P_{i}, i=0,1, \ldots, \mathrm{N}_{r}
$$

where $P_{i}$ is the PER requirements for the $i$-th transmission and $N_{r}$ is the maximum 
retransmission times allowed. Substituting the PER expression into (5), the SNR thresholds for transmission modes combined with Type-I and Type-II HARQ can be obtained by

$$
\gamma_{n, i}=\frac{1}{g_{n, 0}(i+1)} \ln \left(\frac{a_{n, o}}{P_{i}}\right), n=1,2, \ldots, M,
$$

and

$$
\gamma_{n, i}=\frac{1}{g_{n, i}} \ln \left(\frac{a_{n, i}}{P_{i}}\right), n=1,2, \ldots, M
$$

respectively.

To avoid computation complexity, a simple dynamic threshold switching method is proposed in [18] by letting the PER condition in each retransmission to be equal, i.e.,

$$
P_{i}=P_{\text {loss }}^{1 /\left(N_{r}+1\right)},
$$

The correponding SNR thresholds for transmission modes combined with Type I HARQ and Type - II HARQ are

$$
\begin{array}{r}
\gamma_{n, i}=\frac{1}{g_{n, 0}(i+1)} \ln \left(\frac{a_{n, 0}}{P_{\text {loss }}^{1 /\left(N_{r}+1\right)}}\right), n=1,2, \ldots, M \\
\gamma_{n, i}=\frac{1}{g_{n, i}} \ln \left(\frac{a_{n, i}}{P_{\text {loss }}^{1 /\left(N_{r}+1\right)}}\right), n=1,2, \ldots, M
\end{array}
$$

\subsection{The Proposed Dynamic Transmission-Mode Selection Scheme}

The existing work in Section 3.1 on dynamic thresholds switching assumes the same PER requirements, $P_{i}$, for different retransmission time $i$. In this work, we remove this constraint by only limiting the overall PER of each packet transmission, and no longer require each PER condition to be the same, i.e, the SNR shresholds are derived directly from

$$
\prod_{i=0}^{N_{r}} \operatorname{PER}_{n, i}(\gamma) \leq \prod_{i=0}^{N_{r}} P_{i}=P_{\text {loss }} .
$$

Substisuting the PER expressions in (4) and (5) into the (12), we have

$$
\prod_{i=0}^{N_{r}} a_{n, 0} e^{-g_{n, 0}(i+1) \gamma_{n}} \leq P_{\text {loss }}, n=1,2, \ldots, M
$$

for Type-I HARQ and

$$
\prod_{i=0}^{N_{r}} a_{n, i} e^{-g_{n, i} \gamma_{n}} \leq P_{\text {loss }}, n=1,2, \ldots, M
$$

for Type-II HARQ.

Therefore we can develop new SNR threshods for tranmission mode selection combined with Type-I HARQ and Type-II HARQ , which are

$$
\gamma_{n}=\frac{1}{g_{n, 0} \sum_{i=0}^{N_{r}}(i+1)} \ln \left(\frac{a_{n, 0}^{N_{r}+1}}{P_{\text {loss }}}\right), n=1,2, \ldots, M
$$


and

$$
\gamma_{n}=\frac{1}{\sum_{i=0}^{N_{r}} g_{n, i}} \ln \left(\frac{\prod_{i=0}^{N_{r}} a_{n, i}}{P_{\text {loss }}}\right), n=1,2, \ldots, M
$$

receptively.

The advantage of the proposed dynamic Transmission-mode selection scheme can be explained from the schematic PER curve in Fig. 2. The convenetional threshold calculationg scheme using (4) requires $\operatorname{PER}_{n, 0}(\gamma) \leq P_{\text {loss }}$, and threshold value $\gamma_{\text {con }}$ can be obtained. The basic idea of the proposed scheme is to constrain the overall PER to satify $\prod_{i=0}^{N_{r}} P_{i} \leq P_{\text {loss }}$, which means in Fig. 2 when $P_{n, 0} \cdot P_{n, 1} \cdot P_{n, 2} \leq P_{\text {loss }}$, the threshold $\gamma_{\text {agg }}$ can be obtained. Considering $P_{n, i} \geq P_{\text {loss }}$ for any $i, \gamma_{\text {agg }}$ must be less than $\gamma_{\text {con }}$, which is also revealed in the figure. Due to the fact that larger PER torelence yields high modulation order in AMC, this implies that the proposed dynamic TM selection scheme is prior to select high order modulation patterns, which leads to high spectrum efficiency.

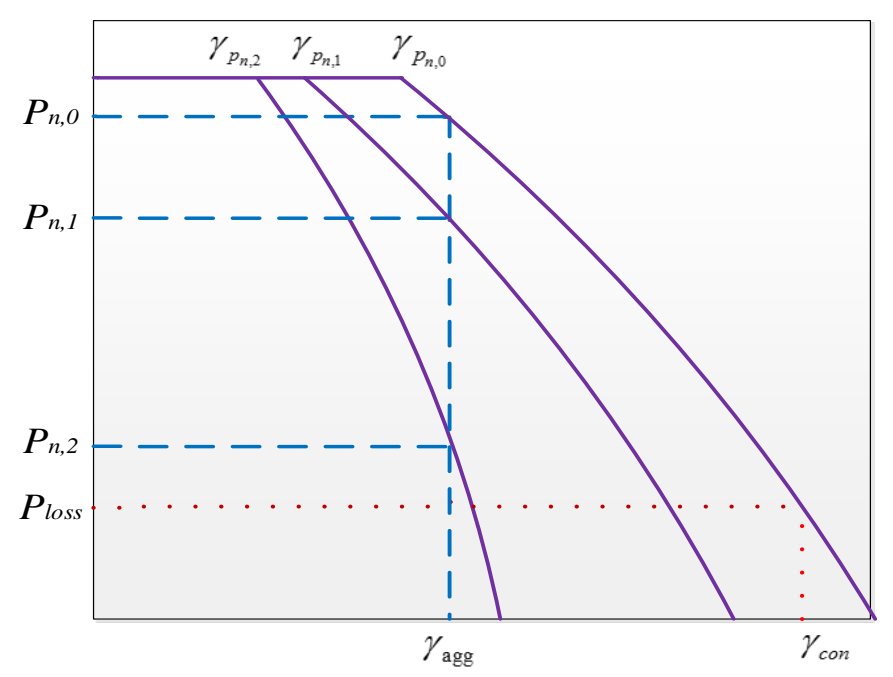

Fig. 2. PER for different transmission modes combined with HARQ

The proposed scheme can be performed in the following steps. First, according to the type of hybrid ARQ (Type-I or Type-II), the initial threshold $\gamma_{n}$ for each transmission mode is calculated according to (15) or (16) with a given QoS requirement $P_{\text {loss }}$; Second, we compare this initial threhold with the minmum applicable SNR threshold $\gamma_{p_{n, i}}$ for each transmission mode. If $\gamma_{n} \geq \gamma_{p_{n, i}}$, the threshold $\gamma_{\mathrm{agg}}$ will be used for all retransmissions of the onging packet, and the threshold of each transmission mode will keep the same value, i.e., $\gamma_{\mathrm{agg}}$; If $\gamma_{n}<\gamma_{p_{n, i}}$, 
we apply $\gamma_{p_{n, i}}$ as the current threshold but set the PER of the current transmission to 1 , and continue to calculate the thresholds for the rest retransmissions using (15) or (16) untill all the threshold in each model and every transmission is greater than $\gamma_{p_{n, i}}$. It is interesting that, in this case, the threshold in each retrasmission of the same transmission mode could be different.

For clarity, we further provide an example to illustrate the threshold calculation steps of the proposed scheme. As an example, when $\mathrm{SNR}=12 \mathrm{~dB}, P_{\text {loss }}=10^{-6}, \mathrm{Nr}=2$, combined with Type - II HARQ, the threshold of TM mode 1 can be calculated according to (16): $\gamma_{1}=0.5827$, and at this time we can obtain $\gamma_{p_{1,0}}=0.7565, \gamma_{p_{1,1}}=0.3864, \gamma_{p_{1,2}}=0.2541$. It can be observed that $\gamma_{1}$ is less than $\gamma_{p_{1,0}}$, but greater than $\gamma_{p_{1,1}}$ and $\gamma_{p_{1,2}}$. For this case, we can set $\gamma_{1,0}$ to $\gamma_{p_{1,0}}$, $\gamma_{1,1}=\gamma_{1,2}=\frac{1}{g_{1,1}+g_{1,2}} \ln \left(\frac{a_{1,1} \cdot a_{1,2}}{P_{\text {loss }}}\right)=0.5478$. In this way, it can be ensured that each settig threshold is no less than its required threshold, meaning the PER requirement, is well met. Hence the SNR thresholds of TM mode 1 for the 3 (re)transmissons are 0.7565, 0.5478, 0.5478, respectively.

\subsection{Performance Study}

\subsubsection{Signal-antenna case}

If only signal antenna is equipped in the transmitter and receiver, the performance of the proposed scheme can be evaluated following the same way as in [18]. According to the PDF of the received SNR in Nakagami block fading channel, the probability of selecting TM mode $n$ in the $i$-th (re)transmission is:

$$
P r_{n, i}=\int_{\gamma_{n, i}}^{\gamma_{n+1, i}} p_{\gamma}(\gamma) d \gamma=\frac{\Gamma\left(m, \frac{m \gamma_{n, i}}{\bar{\gamma}}\right)-\Gamma\left(m, \frac{m \gamma_{n+1, i}}{\bar{\gamma}}\right)}{\Gamma(m)}
$$

where $\Gamma(m, x)=\int_{x}^{\infty} t^{m-1} e^{-t} d t$ is the complementary incomlete Gamma funciton.

Thus, the average PER of the $i$-th transmission can be expressed by:

$$
\overline{\operatorname{PER}}_{i}=\frac{\sum_{n=1}^{M} R_{n} \operatorname{Pr}_{n, i} \overline{P E R}_{n, i}}{\sum_{n=1}^{M} R_{n} \operatorname{Pr}_{n, i}}
$$

where $\overline{P E R}_{n, i}$ is the average PER of selecting transmission mode $n$ in the $i$-th transmission combined with Type-I HARQ and Type - II HARQ ${ }^{[1]}$, and $R_{n}$ is defined in Table $\mathbf{1}$.

Since for the single-antenna case, the difference of the average transmission delay and spectrum efficiency completely depend on the SNR threshold, in this sub-section we directly obtain the average delay and spetrum efficiency through (17) and the results in [18].

The average time delay rate is

$$
E(\bar{D})=\left[1+\sum_{\mathrm{i}=0}^{N_{r}-1} \prod_{j=0}^{i} \overline{P E R}_{j}\right] T
$$

where $T$ repersents the time duration of one successful transmission, which is the product of 
HARQ retransmission times and TTI duration time.

And average spectral efficiency can be obtained:

$$
\overline{S E}=\frac{1}{\bar{N}} \sum_{i=0}^{N_{r}}\left(P_{i}^{N} \overline{S E}_{i}\right)
$$

where $P_{i}^{N}$ denotes the probability that the onging transmission of a packet is the $i$-th retransmission, $\bar{N}$ is the average retransmission time, and $\overline{S E}_{i}$ is the spectrum efficiency of the $i$-th retransmission, both are omitted in this paper since they can be found in [18] .

\subsubsection{MIMO Case}

When the transmitter and receiver are equipped with multiple antennas, the distrubtion of received SNR should be reconsidered. In this work we assume space-time block coding is applied for multi-antenna transmissions as in $[19,20]$, hence the PDF of the received SNR can be expressed by

$$
\mathrm{p}_{\gamma}(\gamma)=\frac{\gamma^{m \kappa-1}}{\Gamma(m \kappa)}\left(\frac{m n_{T} R_{c}}{\bar{\gamma}}\right)^{m \kappa} e^{-\frac{m n_{T} R_{c}}{\bar{\gamma}} \gamma}, \gamma \geq 0
$$

where $\kappa=\mathrm{n}_{T} \cdot \mathrm{n}_{R}$, and $R_{c}$ is the code rate of space-time coding.

Compared to (17) for single-antenna case, the probability of selecting TM mode $n$ in the $i$-th (re)transmission is changed into :

$$
P r_{n, i}=\int_{\gamma_{n, i}}^{\gamma_{n+1, i}} p_{\gamma}(\gamma) d \gamma=\frac{\Gamma\left(m \kappa, m n_{T} R_{c} \frac{\gamma_{n+1}}{\gamma}\right)-\Gamma\left(m \kappa, m n_{T} R_{c} \frac{\gamma_{n}}{\bar{\gamma}}\right)}{\Gamma(m)}
$$

and the average PER of pattern $n$ in the $i$-th transmission for Type-I HARQ and Type-II HARQ, respectively:

$$
\begin{aligned}
\overline{P E R}_{n, i} & =\frac{1}{\operatorname{Pr}_{n, i}} \int_{\gamma_{n, i}}^{\gamma_{n+1, i}} P E R_{n, i}(\gamma) p_{\gamma}(\gamma) d \gamma \\
& =\frac{1}{\operatorname{Pr}_{n, i}} \frac{a_{n, o}}{\Gamma(m)}\left(\frac{m n_{T} R_{c}}{\bar{\gamma}}\right)^{m \kappa} \frac{\Gamma\left(m \kappa, b_{n} \gamma_{n}\right)-\Gamma\left(m \kappa, b_{n} \gamma_{n+1}\right)}{\left(b_{n, i}\right)^{m \kappa}} \\
\overline{P E R}_{n, i} & =\frac{1}{\operatorname{Pr}_{n, i}} \int_{\gamma_{n, i}}^{\gamma_{n+1, i}} P E R_{n, i}(\gamma) p_{\gamma}(\gamma) d \gamma \\
& =\frac{1}{\operatorname{Pr}_{n, i}} \frac{a_{n, i}}{\Gamma(m)}\left(\frac{m n_{T} R_{c}}{\bar{\gamma}}\right)^{m \kappa} \frac{\Gamma\left(m \kappa, b_{n} \gamma_{n}\right)-\Gamma\left(m \kappa, b_{n} \gamma_{n+1}\right)}{\left(b_{n, i}\right)^{m \kappa}}
\end{aligned}
$$

where $b_{n, i}=m n_{T} \mathrm{R}_{c} / \bar{\gamma}+g_{n, 0}(i+1)$ in (23) and $b_{n, i}=m n_{T} \mathrm{R}_{c} / \bar{\gamma}+g_{n, i}$ in (24).

The average time delay rate $E(\bar{D})$ and the average spectral efficiency $\overline{S E}$ can be derived through a similar way as the single-antenna case in Section 3.3.1. The analysis above shows that, the proposed dynamic transmission-mode selection scheme can be readily applied in 
MIMO communication, which is more practical in modern mobile communication systems. In this way, the proposed transmission-mode selection scheme can be analysed and applied in both signal-antenna and MIMO communication scenarios.

\section{Numerical Results}

To reveal the performnace gain of the proposed scheme, in this section we evaluated the average spectrum efficiency and transmission delay in an AMC/Type-2 HARQ wireless networks. The conventional scheme (displayed as 'conventional scheme' in the figures) and an existing cross-design scheme using dynamic switching threshold proposed in [18] (displayed as 'dynamic switching' in the figures), which have been descripted in Section 3.1, are also simulated for comparsion. Without loss of generallity, the simulations were perfomed assuming Rayleigh channel (a special case, $m=1$, of Nakigami channel) as in [14][18] for comparision. And, throughout this section, $\mathrm{P}_{\text {tgt }}$ is used to denote the overall tolerable packet error rate for each message packet, which corresponds to the QoS requirement from the upper layers.

Fig. 3 shows the average spectral efficiency with different PER requirements $P_{t g t}$ for $\mathrm{SNR}=12 \mathrm{~dB}$.

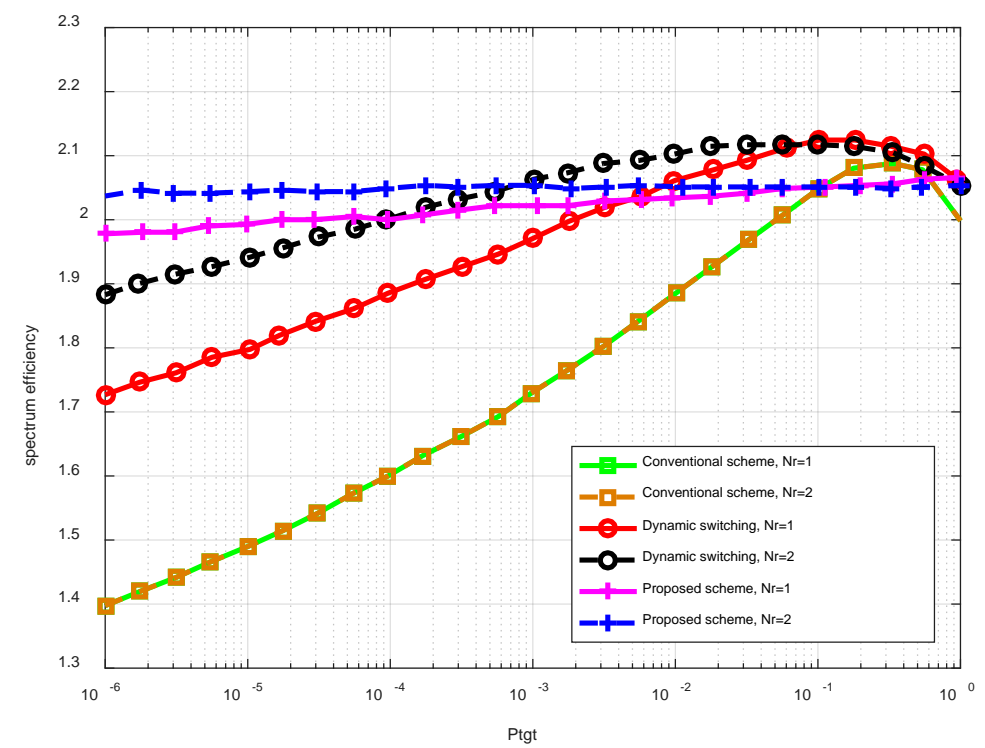

Fig. 3. Average spectral efficiency with different requirements of PER for SNR=12dB

As is shown in Fig. 3, the proposed transmission-mode selection scheme achieves high average spectrum efficiency, especially for small $\mathrm{P}_{t g t}$. Specifically, For $N_{\mathrm{r}}=1, \mathrm{P}_{t g t}$ ranges from $10^{-6}$ to $10^{-2.25}$, and $N_{\mathrm{r}}=2$ and $\mathrm{P}_{\text {tgt }}$ ranges from $10^{-6}$ to $10^{-3.25}$, the proposed scheme shows much higher average spectral efficiency than the conventional and dynamic switching schemes in [18].

Moreover, when $\mathrm{SNR}=12 \mathrm{~dB}$, the average spectral efficiency of the proposed scheme changes with $\mathrm{P}_{\text {tgt }}$ more steadily than the dynamic threshold scheme. This is reasonable since 
the probability of $\gamma_{n}>\gamma_{p_{n, i}}$ becomes higher with the increase of retransmission time $i$, which leads to a slow change on spectrum efficiency.

It can also be oberserved from Fig. 3 that $N_{\mathrm{r}}$ has negligible influence on the average spectral efficiency for the conventional scheme. This is because retransmissions nearly happen due to the strict PER requirements from (4), which results in low modulation coding rate i.e., low spectrum efficiency.

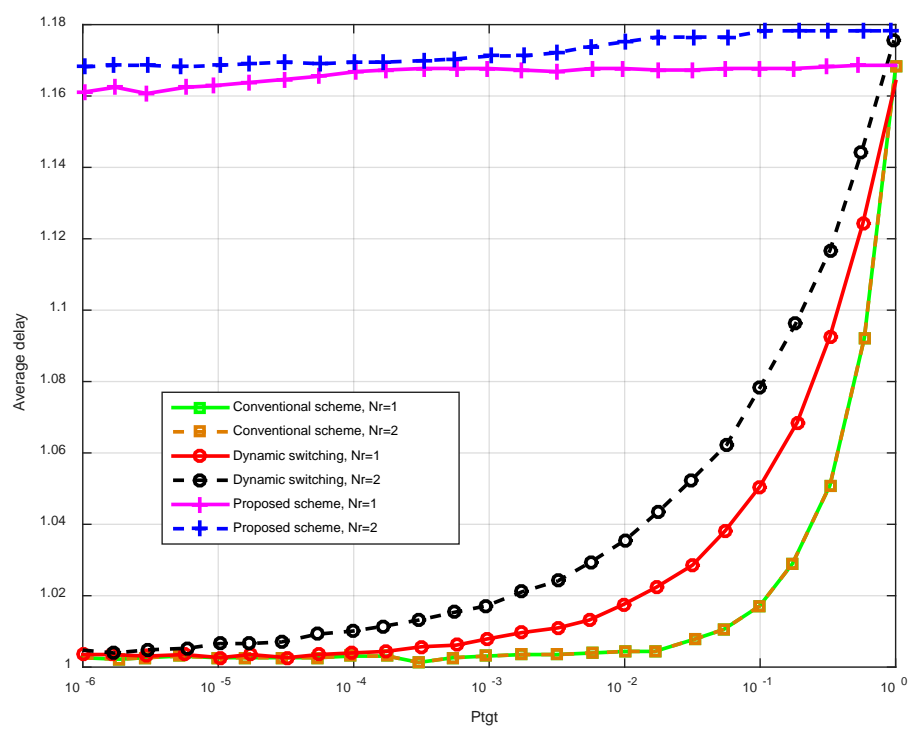

Fig. 4. Average time delay with different requirements of PER for SNR=12dB.

Fig. 4 shows the average delay of different schemes when $S N R=12 d B$. It can be oberserved that the average delay of the proposed scheme is the largest, this is a price that we use higher modulation coding rate (higher TM mode $n$ ) for higher transmission rate. Even though, as is shown in Fig. 3, a small increase in average delay acommpanies with improvement in specturm efficency, especially for small $\mathbf{P}_{t g t}$.

To further reveal the performance gain of the proposed scheme, Fig. 5 and Fig. 6 show the average spectral efficiency and the delay of each scheme with different PER requirement for SNR $=4 \mathrm{~dB}$. It is observed from Fig. 5 and Fig. 6 that the smaller the SNR is, i.e. the worse the quality of the channel is, the better the scheme put forward in the paper will be. When $\mathrm{SNR}=4 \mathrm{~dB}$, within the whole scope of $\mathrm{P}_{t g t}$, the average spectral efficiency of the improvement scheme in the paper is on the rise, and higher than all the others. 


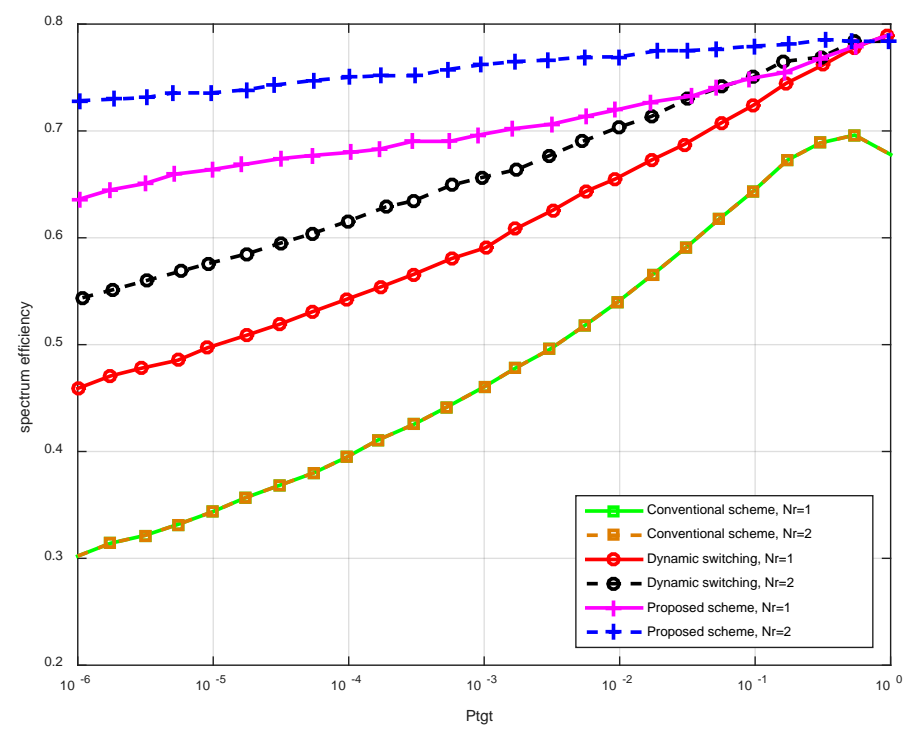

Fig. 5. Average spectral efficiency with different $P E R$ requirements for $S N R=4 d B$.

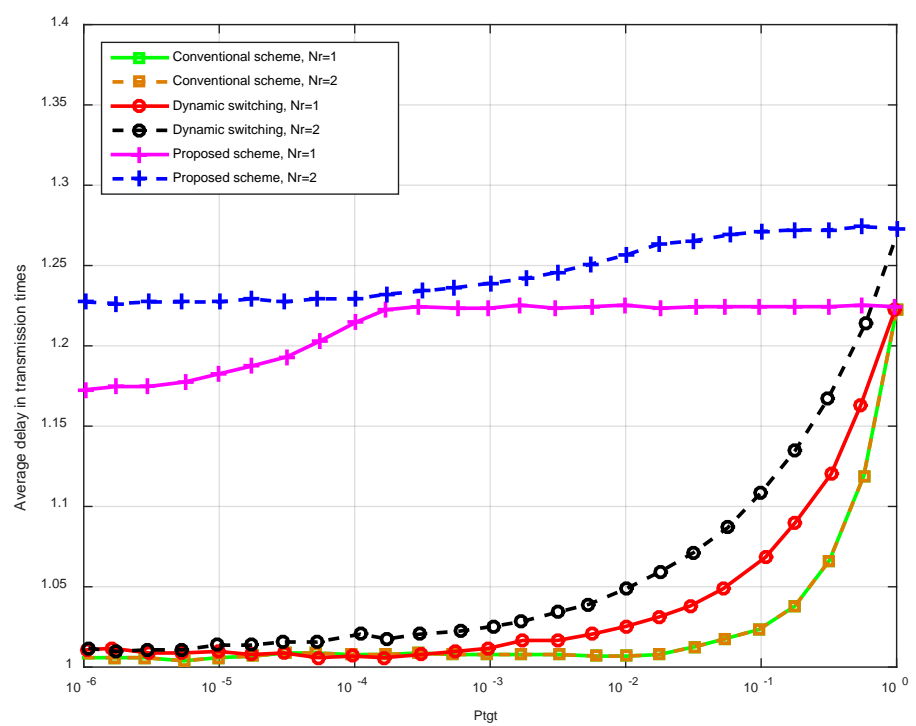

Fig. 6. Average time delay with different $\mathrm{PER}$ requirements for $\mathrm{SNR}=4 \mathrm{~dB}$.

Fig. 7 shows the average spectral efficiency of the three schemes with the received SNR. As is shown in Fig. 7 , the proposed tranmssion mode seleciton scheme performs better in both $\mathrm{P}_{\mathrm{tgt}}=0.01$ and $\mathrm{P}_{\mathrm{tgt}}=10^{-4}$ cases, especially when $\mathrm{P}_{\mathrm{tgt}}=0.01$. Another observation is that, when $\mathrm{P}_{\mathrm{tgt}}=0.01$, the average spectral efficiency of all the three schemes are higher than that when $\mathrm{P}_{\mathrm{tgt}}=10^{-4}$, while tends to be the same as SNR increases, which is a result of very low bit error rate. 


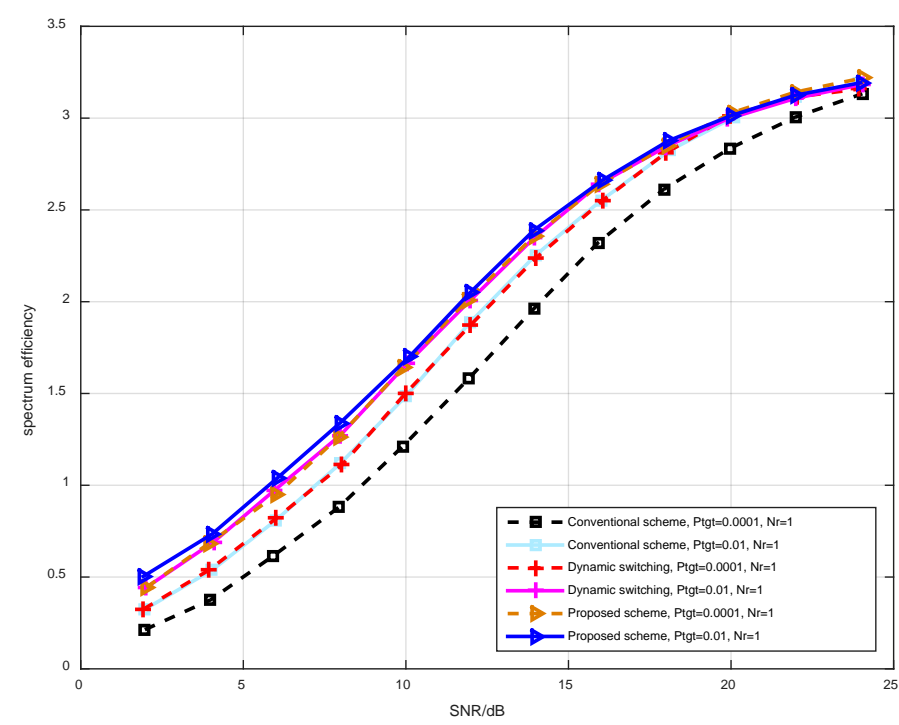

Fig. 7. Average spectral efficiency with different received SNR

Fig. 8 and Fig. 9 shows the probability of TM mode 1,4 and 5 being selected. As can be seen, for a given SNR, the proposed scheme is more likely to select transmission mode with higher rate. Specifically, it can be observed in the two figures that, when SNR is small, e.g., ranging from $-10 \mathrm{~dB}$ to $0 \mathrm{~dB}$, the probablity of selecting TM 1 for the proposed scheme is the highest. This is because the state of the channel is undergoing deep fading, thus the other two schemes are more likely not to to transmit, making the probabilty of selecting TM 1 for the other scheme is lower than the proposed scheme. On the other hand, when SNR is high, say $\mathrm{SNR}=15 \mathrm{~dB}$, the conventional scheme and the dynamic switching scheme are more likely to select TM 4 than the proposed scheme, because in this case the proposed improvement scheme is more likely to select TM 5, which obviously acheives higher transmission rate.

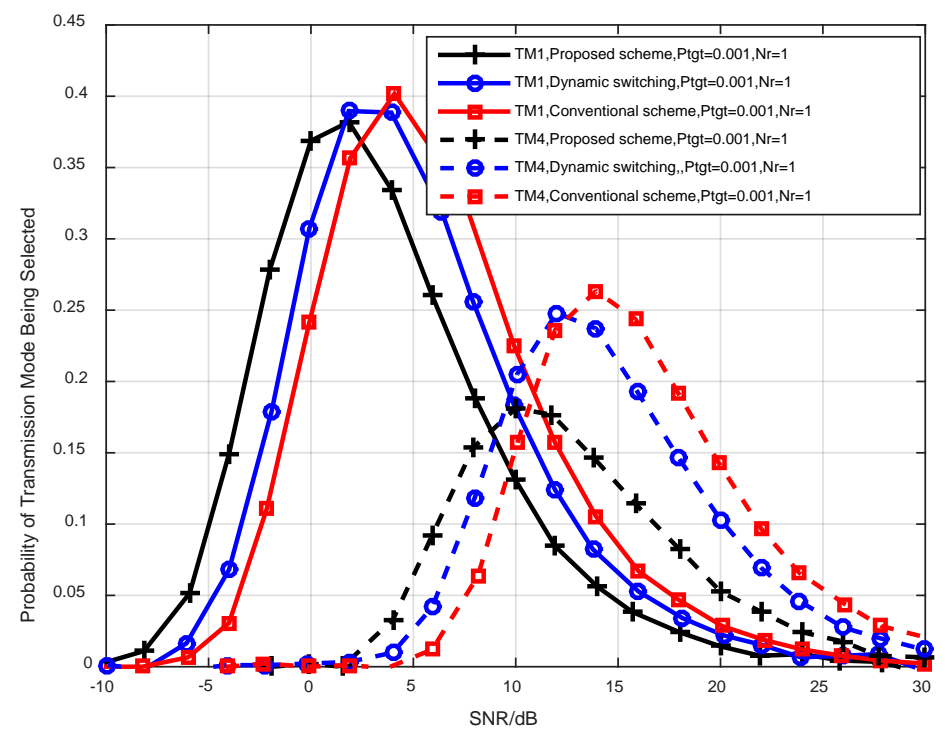

Fig. 8. Probability of the transmission mode (TM) 1 and 4 being selected. 


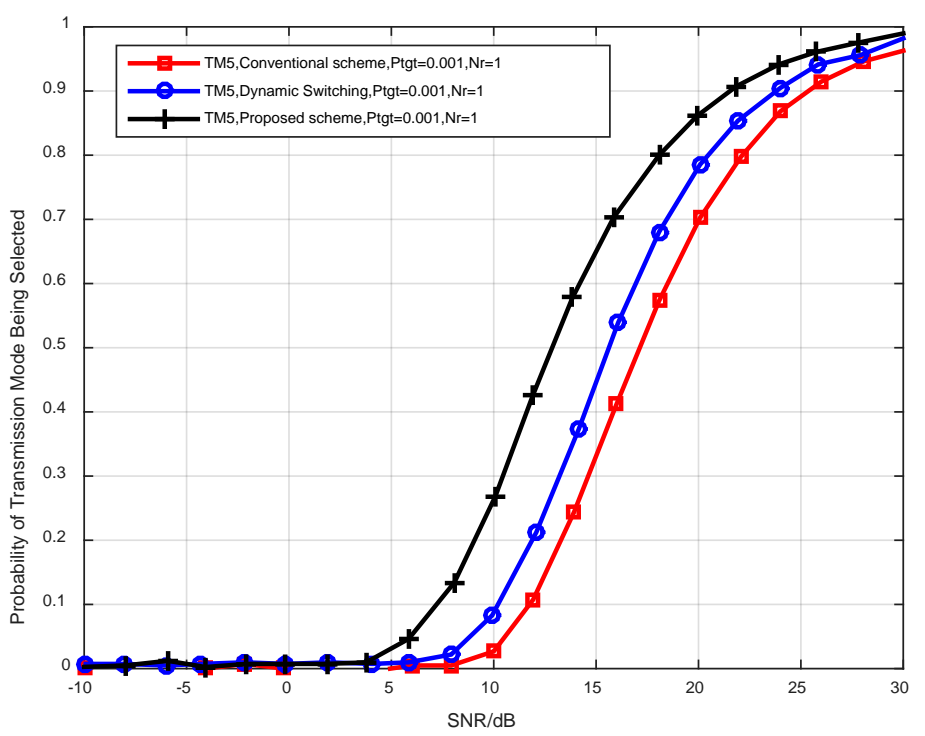

Fig. 9. Probability of the transmission mode (TM) 5 being selected.

Fig. 10 reveals the average spectral efficiency in MIMO scenarios when $S N R=4 d B$ and $N_{r}$ $=1$. For comparsion, we also extend the dynamic switching scheme to MIMO scenarios. From Fig. 10 it can be observed that the proposed scheme generally acheives higher average spectrum efficiency than the dynamic switching scheme for high QoS requirements (small $P_{\mathrm{tgt}}$ ). When the number of transmitting antennas $n_{T}$ is fixed, the larger the number of receiving antennas $n_{R}$ yields larger average spectral efficiency.

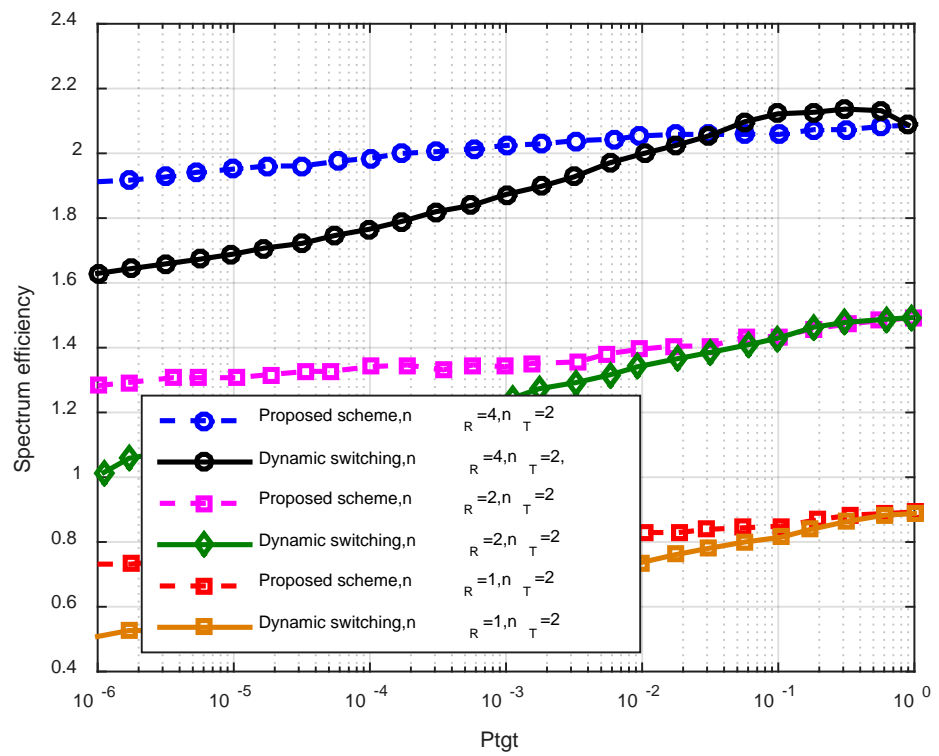

Fig 10. Average spectral efficiency in $\mathrm{MIMO}$ case for $\mathrm{SNR}=4 \mathrm{~dB}$ and $N_{\mathrm{r}}=1$.

Fig. 11 shows the change of the average spectral efficiency with the numbers of receiving antennas and the maximum transmsision times $N_{\mathrm{r}}$ when $\mathrm{SNR}=4 \mathrm{~dB}$ in the proposed scheme. It is confirmed that when the number of transmitting antennas $n_{T}$ is fixed, larger the number of 
receiving antennas $n_{R}$ means larger average spectral efficiency. It is also revealed in Fig. 11 that the maximum number of retransmission can bring system performance gain for any given numbers of receiving antennas.

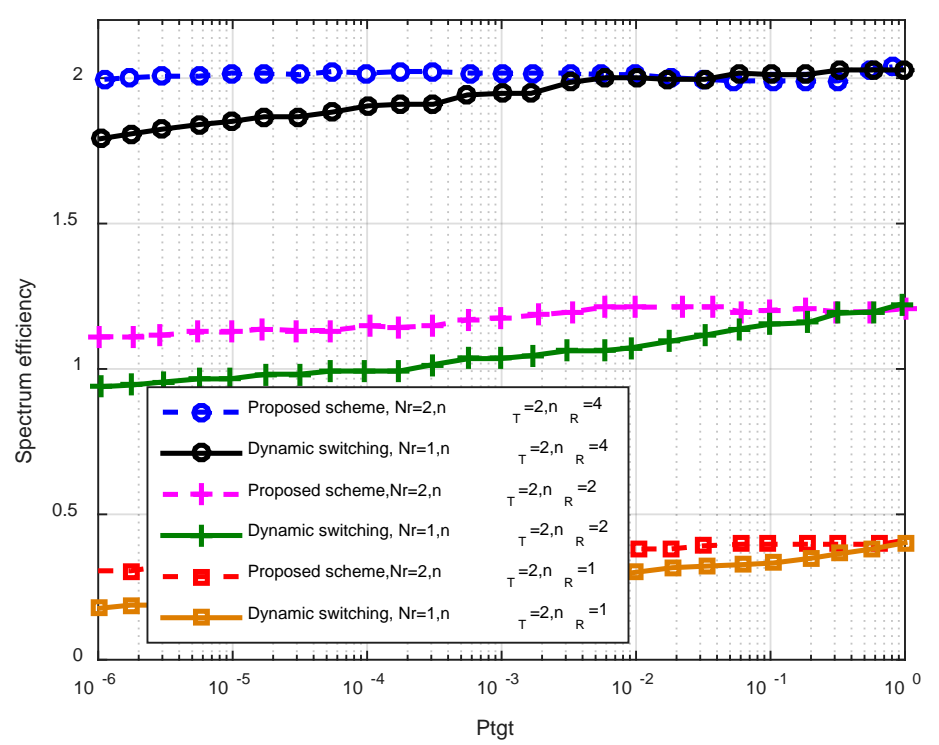

Fig. 11. Average spectral efficiency of the proposed scheme with the change of $n_{R}$ and $N_{\mathrm{r}}$.

Fig. 12 provides the performance comparision in average spectrum efficiency of the proposed and dynamic switching scheme in [18]. As can be seen from Fig. 12, the proposed scheme achieves a higher average spectrum efficiency for the low SNR region, which is accord with the discussion in this paper. It is also revealed that, when the number of transmission antennas is fixed, the large the number of receiving antennas leads to the higher average spectrum efficiency.

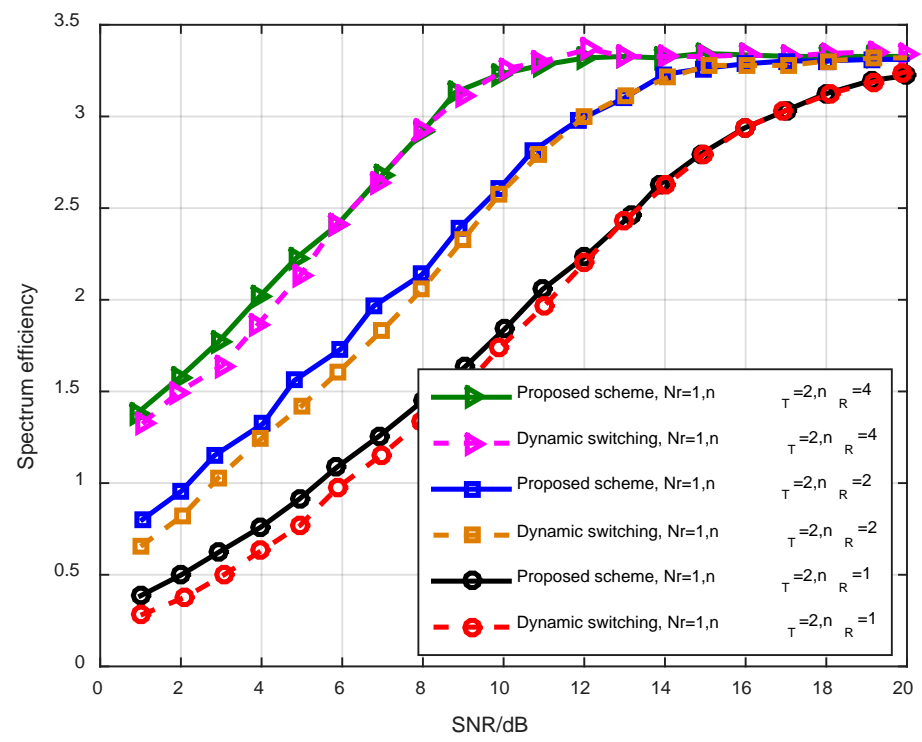

Fig. 12. Average spectral efficiency with the received SNR, $P_{\mathrm{tgt}}=10^{-3}$. 


\section{Conclusion}

In this paper, the cross-layer design of AMC and HARQ is studied, and a new dynamic transmission-mode selection scheme to improve system spectrum efficiency was developed. Compared with conventional and the existing dynamic scheme for transmission mode selection, the proposed scheme is designed to dynamically select transmission mode for each packet transmission and retransmission, and be more inclined to apply higher modulation rate in the first several retransmissions, thus can achieve more flexibility in the selection of modulation coding rate, which corresponds to higher spectrum efficiency.

\section{References}

[1] JF Hayas, "Adaptive feedback Communications," IEEE Transactions Communication Technology, 16(1):29-34, 1968. Article (CrossRef Link)

[2] JK Cavers, "Variable-rate transmission for Reyleigh fading channelsm," IEEE Transactions Communication Technology, 20(1):15-22, 1972. Article (CrossRef Link)

[3] D. Goeckel, "Adaptive coding for time-varying channels using outdated fading estimates," IEEE Trans. Commun., vol. 47, no. 6, pp. 844-855, Jun. 1999. Article (CrossRef Link)

[4] S Zhou, K Zhang, Z Niu,et al., "Queuing analysis on MIMO systems with adaptive modulation and coding," in Proc. of IEEE International Conference on Communicaitons, 3400-3405, 2008.8. Article (CrossRef Link)

[5] M. Jabi, M. Benjillali, L. Szczecinski, and F. Labeau, "Energy efficiency of adaptive HARQ," IEEE Trans. Commun.,vol. 64, no. 2, pp. 818-831, Feb. 2016. Article (CrossRef Link)

[6] 3GPP, "3GPP TS 36.321 V13.1.0 release 13: LTE; Evolved Universal Terrestrial Radio Access (E-UTRA); Medium access control (MAC) protocol specification,” 2016.03.

[7] 3GPP, "3GPP TS 36.213 V13.1.1 release 13: LTE; Evolved Universal Terrestrial Radio Access (E-UTRA); Physical layer procedures,” 2016.03.

[8] D Mischa, and T Nakamura, 5G Mobile and Wireless Communications Technology, Cambridge University Press, 2016.

[9] R. Sassioui; M. Jabi, L. Szczecinski, L. B. Le; M. Benjillali, B. Pelletier, "HARQ and AMC: Friends or Foes?" IEEE Transactions on Communications, vol.99, no.99, pp.1-10, 2016. Article (CrossRef Link)

[10] V Srivastava, M Motani, “Cross-layer design: a survey and the road ahead," IEEE Communications Magazine, 43(12):112-119, 2006. Article (CrossRef Link)

[11] B Fu, Y Xiao, H J Deng, et al., "A Survey of Cross-Layer Designs in Wireless Networks,” IEEE Communications Surveys \& Tutorials, 16(1):110-126, 2014. Article (CrossRef Link)

[12] Q Liu, S Zhou, G B Giannakis, "Cross-layer combining of adaptive modulation and coding with truncated ARQ over wireless links,” IEEE Transactions Wireless Communications, 3(5): 1746-1755, 2004. Article (CrossRef Link)

[13] X Wang, Q Liu, G B Giannakis, "Analyzing and optimizing adaptive modulation coding jointly with ARQ for Qos-guaranteed traffic,” IEEE Transactions Vehicular Technology, 56(2):710-720, 2007. Article (CrossRef Link)

[14] J Ramis, G Femenias, "Cross-layer design of zdaptive multirate wireless networks using truncated HARQ,” IEEE Transactions Vehicular Technology, 60(3):944-954, 2011. Article (CrossRef Link)

[15] H Si, W K Jin, C G Kang, "Design of Adaptive Modulation and Coding Scheme for Truncated HARQ,” International Symposium on Wireless Pervasive Computing, IEEE, 269-280, 2007. Article (CrossRef Link)

[16] R Cohen, G Grebla, L Katzir, "Cross-layer hybrid FEC/ARQ reliable multicast with adaptive modulation and coding in broadband wireless networks," IEEE/ACM Transactions Networking, 18(6):1908-1920, 2010. Article (CrossRef Link)

[17] D Wang, L Li, Y Fang, et al., "Cross-Layer Design for LTE System with Jointly AMC and ARQ on Fading Channel," in Proc. of IEEE Wireless Communications and Networking Conference 
(WCNC), 5:356-361, 2013. Article (CrossRef Link)

[18] P Zhang, Y Miao, and Y Zhao, "Cross-layer design of AMC and Truncated HARQ using Dynamic switching thresholds,” in Proc. of IEEE Wireless Communications and Networking Conference (WCNC2013), 906-911, 2013. Article (CrossRef Link)

[19] A Maaref, S Aissa, "Combined adaptive modulation and truncated ARQ for packet data transmission in MIMO systems," in Proc. of IEEE Global Telecommunications Conference, 6:3818-3822, 2004. Article (CrossRef Link)

[20] X Zhu, D Yuan, “Cross-layer design for MIMO correlated Nakagami fading channels,” IEEE Proceedings of the International Workshop on Cross Layer Design, 50-54, 2011.

Article (CrossRef Link)

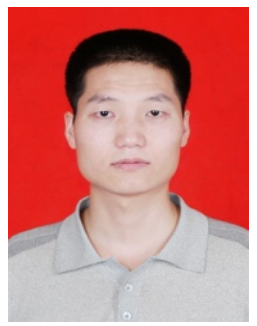

Xiaohui Ma is currently an engineer in Xi'an Research Institute of Surveying and Mapping, Xi'an, China. He research interests include space geodesy, invariant reference point simulation of VLBI, and the wireless communications techniques in GNSS.

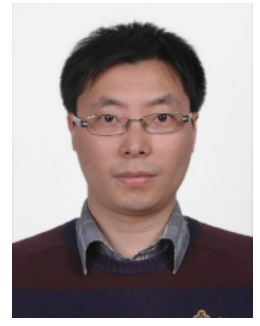

Guobing $\mathbf{L i}$ is currently an Associate Professor in the School of Electronic and Information Engineering, Xi'an Jiaotong University, Xi'an, China. His research interests include wireless relay network, MIMO techniques and 5G mobile communication systems.

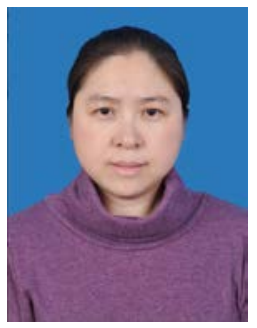

Guomei Zhang is currently an Associate Professor in the School of Electronic and Information Engineering, Xi'an Jiaotong University, Xi'an, China. Her research interests include MIMO, CoMP, Massive MIMO and Interference Management in wireless communication systems and networks. 